\title{
Propriedades psicométricas do WHOQOL-HIV Bref para avaliação da qualidade de vida
}

\author{
Marise Fagundes Silveira ${ }^{1}$ \\ Antônio Carlos Ferreira ${ }^{1}$ \\ Maria Fernanda Santos Figueiredo Brito ${ }^{1}$ \\ Lucinéia de Pinho ${ }^{1}$ \\ Antônio Lúcio Teixeira Júnior² \\ Mariângela Carneiro \\ ${ }^{1}$ Universidade Estadual de Montes Claros, Montes Claros, MG \\ ${ }^{2}$ Universidade Federal de Minas Gerais, Belo Horizonte, $M G$
}

\begin{abstract}
Resumo
Objetivo: Avaliar as propriedades psicométricas do questionário WHOQOL-HIV Bref em pessoas com HIV/AIDS. Método: O instrumento foi aplicado a 226 pessoas com HIV/AIDS do Norte de Minas Gerais. As propriedades psicométricas foram avaliadas pela validade de construto, análise da confiabilidade e validade de critério. Resultados: O modelo com seis domínios apresentou ajustamento aceitável $\left(\chi^{2} / \mathrm{gl}=1,77, \mathrm{p}<0,001, \mathrm{GFI}=0,85, \mathrm{CFI}=0,89\right.$, RMSEA = 0,058). O WHOQOL-HIV Bref apresentou correlações estatisticamente significativas entre os seis domínios, consistência interna e reprodutibilidade satisfatórias. Os escores dos domínios demonstraram correlação significativa com o escore da qualidade de vida geral e dos itens que avaliam a autopercepção da qualidade de vida e da saúde, correlação significativa com o resultado global da depressão e diferenças estatisticamente significativas entre subgrupos de pacientes segundo variáveis socioeconômicas e clínicas. Conclusões: $\mathrm{O}$ WHOQOL-HIV Bref apresentou-se válido, confiável para medir a qualidade de vida de pessoas com HIV/AIDS.

Palavras-chave: Estudos de validação; WHOQOL-HIV-Bref; qualidade de vida; HIV; síndrome da imunodeficiência adquirida.
\end{abstract}

Psychometric properties of the WHOQOL-HIV Bref for quality of life assessment

\begin{abstract}
Objective: To evaluate the psychometric properties of the WHOQOL-HIV Bref questionnaire in people with HIV / AIDS. Method: The questionnaire was applied to 226 people living with HIV/AIDS in the Northern part of the state of Minas Gerais. The psychometric properties were evaluated by construct validity, reliability analysis, and criteria validity. Results: The model with six domains had an acceptable adjustment $\left(\chi^{2} / \mathrm{gl}=1.77, \mathrm{p}<0.001\right.$, GFI $=0.85$, CFI $=0.89$, RMSEA $\left.=0.058\right)$. The WHOQOL-HIV Bref showed statistically significant correlations among the six domains, satisfactory internal consistency and reproducibility. The instrument scores showed a significant correlation with the general quality of life score and the items that assess the self-perception of quality of life and health, a significant correlation with the overall result of depression, and statistically significant differences between subgroups of patients. Conclusions: The WHOQOL-HIV Bref was valid, reliable to measure the quality of life of people with HIV/AIDS.
\end{abstract}

Keywords: Validation Studies; WHOQOL-HIV-Bref; quality of life; HIV; acquired immunodeficiency syndrome

Propiedades psicométricas del WHOQOL-VIH Bref para evaluación de calidad de vida

\begin{abstract}
Resumen
Objetivo: Evaluar las propiedades psicométricas del cuestionario WHOQOL- HIV Bref en personas con HIV/SIDA. Método: El instrumento fue aplicado a 226 personas con HIV/SIDA del Norte de Minas Gerais. Las propiedades psicométricas fueron evaluadas por validez de constructo, análisis de confiabilidad, y validez de criterio. Resultados: El modelo con seis dominios presentó un ajuste aceptable $\left(\chi^{2} / \mathrm{gl}=1,77, \mathrm{p}<0,001, \mathrm{GFI}=0,85, \mathrm{CFI}=0,89\right.$, RMSEA $\left.=0,058\right)$. El WHOQOL-HIV Bref presentó correlaciones estadísticamente significativas entre los seis dominios, consistencia interna, y reproductibilidad satisfactorias. Los resultados de los dominios demostraron correlación significativa con el resultado de calidad de vida general y de los ítems que evalúan la autopercepción de calidad de vida y de la salud, correlación significativa con el resultado global de depresión y diferencias estadísticamente significativas entre subgrupos de pacientes según variables socioeconómicas y clínicas. Conclusiones: El WHOQOL- HIV Bref se presentó como válido y confiable para medir la calidad de vida de personas con HIV/SIDA. Palabras clave: estudios de validación; WHOQOL-HIV-Bref; calidad de vida; HIV; síndrome de inmunodeficiencia adquirida
\end{abstract}

\section{Introdução}

A infecção de indivíduos pelo Vírus da Imunodeficiência Humana (HIV) é a causa da Síndrome da
Imunodeficiência Adquirida (AIDS), que por sua vez constitui um importante problema de saúde pública global (Unaids, 2012; WHO Fact Sheet, 2014). Em resposta ao esforço mundial para o combate do HIV, com 
ações para redução da transmissão, prevenção de novas infecções, avanço dos tratamentos e maior acesso aos mesmos, a incidência do vírus tem diminuído (Unaids, 2012) e a sobrevida dos pacientes aumentado. Com isso, a AIDS passou a ter o status de doença crônica cada vez mais manejável (WHO, 2003). Todavia, o controle do HIV é ainda um desafio para saúde pública, visto o número ainda expressivo de pacientes infectados em muitas partes do mundo (Unaids, 2012).

A infecção por HIV pode ter repercussões físicas, psicológicas, sociais (Reychler, Caty, Vincent, Billo \& Yombi, 2013; WHO, 2003), causando grande impacto na qualidade de vida das pessoas com essa condição. A qualidade de vida é um componente importante para avaliação do bem-estar de pessoas vivendo com o vírus HIV. Por isso, a qualidade de vida desses indivíduos tem sido cada vez mais monitorada, o que é essencial para o direcionamento de ações e intervenções do setor saúde e dos seus profissionais e planejamento de políticas públicas voltadas a essa população (Costa, Oliveira \& Formozo, 2015; Reychler et al., 2013).

Instrumentos válidos e confiáveis para medir a qualidade de vida de pessoas vivendo com HIV/AIDS são necessários (Cooper, Clatworthy, Harding, Whetham, \& Emerge Consortium, 2017; Reychler et al., 2013). A princípio, eram utilizados instrumentos genéricos para avaliar a qualidade de vida, como o World Health Organization Quality of Life (WHOQOL-100) e o WHOQOL-Bref. No entanto, medidas genéricas podem ser menos sensíveis a mudanças na doença ou tratamento do que instrumentos específicos para o HIV (Cooper et al., 2017). Em consequência dos aspectos específicos da população com HIV/AIDS, a Organização Mundial de Saúde desenvolveu um questionário específico para esses indivíduos, o WHOQOL-HIV. Esse instrumento é baseado no WHOQOL-100 e inclui um módulo específico de particular relevância para de pessoas vivendo com HIV/AIDS. Posteriormente, foi elaborada uma versão mais curta do WHOQOL-HIV, o WHOQOL-HIV Bref (WHO, 2003).

O WHOQOL-HIV Bref tem sido usado para a avaliação da qualidade de vida de pacientes que vivem com HIV no cenário internacional (Akinboro et al., 2014; Karkashadze, Gates, Chkhartishvili, DeHovitz, \& Tsertsvadze, 2017; Osei-Yeboah et al., 2017) e nacional (Bellini, Reis, Reinato, Magalhães \& Gir, 2015; Costa \& Oliveira, 2013; Hipolito et al., 2017; Oliveira, Queiroz, Souza, Moura \& Reis, 2017; Quadros et al., 2015). Na análise da qualidade de vida com este instrumento em pessoas infectadas no país, observou-se maior comprometimento dos domínios meio ambiente (Bellini et al., 2015, Costa \& Oliveira, 2013; Hipolito et al., 2017; Oliveira et al., 2017; Quadros et al., 2015), relações sociais (Bellini et al., 2015; Costa \& Oliveira, 2013) e nível de independência (Hipolito et al., 2017; Oliveira et al., 2017; Quadros et al., 2015).

Apesar do WHOQOL-HIV Bref ser bem difundido, ainda são poucos os estudos que avaliam suas propriedades psicométricas (WHO, 2003). No Brasil, na validação do WHOQOL-HIV Bref, o instrumento mostrou-se com características satisfatórias de confiabilidade, validade de construto, validade discriminante e concorrente (Zimpel \& Fleck, 2007). Verificou-se que os valores de alfa de Cronbach foram acima de 0,70 em 27 das 31 facetas do WHOQOL-HIV. Melhores escores de QV ocorreram nos estágios iniciais da infecção, enquanto o grupo AIDS apresentou piores escores em todos os domínios do WHOQOL-HIV, com diferenças estatisticamente significativas nos estágios iniciais. A correlação entre os domínios e a QV geral foi estatisticamente significativa $(r>0,5 ; p<0,01)$ (Zimpel \& Fleck, 2007).

Considerando que a confiabilidade e validade dos instrumentos devem ser confirmadas para cada cultura onde eles são aplicados, o objetivo do presente estudo foi avaliar as propriedades psicométricas do questionário WHOQOL-HIV Bref em uma população da região Norte de Minas Gerais.

\section{Métodos}

O estudo foi aprovado pelo Comitê de Ética da Universidade. Todos os participantes assinaram um Termo de Consentimento"

\section{Participantes}

O presente estudo, autorizado pela coordenação do programa municipal de DST/AIDS de Montes Claros, foi realizado em pacientes atendidos em dois centros de referencia especializada deste município: Centro de Referência de Doenças Infecciosas de Montes Claros e Centro Ambulatorial de Especialidades Tancredo Neves. O tamanho amostral mínimo de 145 indivíduos foi calculado considerando-se as premissas para tamanho amostral em estudo de validação psicométrica, que prevê cinco respondentes para cada item do instrumento (Hair Jr, Black, Babin, Anderson \& Tatham, 2009).

A partir de uma lista contendo 521 pacientes atendidos nos centros de referencia, fornecida pelos seus 
coordenadores, foram selecionados por amostragem aleatória simples 229 indivíduos. Os critérios de exclusão adotados foram: ter idade inferior a 18 anos e ter comprometimento cognitivo.

Participaram do estudo 226 indivíduos adultos vivendo com $\mathrm{HIV} /$ Aids, dos quais 51,8\% eram homens, e a média de idade dos participantes foi de 44 anos. Quanto à escolaridade, 16,4\% eram analfabetos, 35,4\% tinham cursado apenas a educação primária, 16,4\% haviam completado o ensino fundamental, 25,7\% tinham o ensino médio e apenas $6,2 \%$ o curso superior. Em relação à renda individual, $56,3 \%$ recebiam até um salário mínimo e 20,9\% entre 1,1 e 2 salários mínimos, $11,7 \%$ entre 2,1 e 3 salários e $11,2 \%$ superior a 3 salários mínimos. De acordo com o critério de classificação econômica Brasil ${ }^{19}, 8,0 \%, 54,9 \%$ e $37,2 \%$ pertenciam às classes $\mathrm{B}, \mathrm{C}$ e $\mathrm{D}$, respectivamente. Verificou-se que 9,9\% dos pacientes estavam com menos de dois anos de infecção, 24,2\% entre dois a quatro anos de infecção, $23,8 \%$ entre cinco e oito anos de infecção e $42,1 \%$ mais de oito anos.

\section{Instrumentos}

A pesquisa utilizou um questionário estruturado que contemplou as variáveis: sociodemográficas (idade, sexo, escolaridade, renda e classe socioeconômica), clínica (tempo de infecção pelo HIV, contagem de células $\mathrm{CD}^{+}$, presença de febre, perda de peso e presença de diarréia) e comportamental (uso de drogas ilícitas) e os instrumentos WHOQOL-HIV Bref e o Inventário de Depressão de Beck (BDI).

\section{WHOQOL-HIV Bref}

A versão em português do instrumento WHOQOL-HIV Bref é composta por 29 itens que avaliam seis domínios da qualidade de vida relacionada à saúde (Físico, Psicológico, Nível de Independência, Relações Sociais, Ambiente e Espiritualidade) e a qualidade de vida geral. $\mathrm{O}$ instrumento possui ainda duas questões que examinam a auto-percepção da qualidade de vida e da saúde. Os itens são avaliados por meio de uma escala tipo likert, com valores entre um e cinco (WHO, 2002; Zimpel \& Fleck, 2007). Em estudo de confiabilidade e validade, este instrumento demonstrou propriedades psicométricas aceitáveis de consistência interna (com alfa de Cronbach variando de 0,61 a 0,80) e validade de construto, cuja análise fatorial confirmatória indicou ajustamento aceitável, confirmando a estrutura fatorial composta por seis domínios (Canavarro \& Pereira, 2011).
Inventário de Depressão de Beck (BDI)

O Inventário de Depressão de Beck (BDI) é utilizado mundialmente para detectar sintomas depressivos. É uma escala de auto-avaliação composta por 21 itens, cada um com quatro assertivas com pontuação de 0 a 3 que medem a intensidade dos sintomas depressivos (Beck \& Steer, 1996). A versão em português do BDI demonstrou que o instrumento é homogêneo e estável com evidências de validade de critério, concorrente e discriminante (Gomes-Oliveira, Gorenstein, Lotufo Neto, Andrade \& Wang, 2012).

\section{Procedimentos}

Os pacientes foram entrevistados nos serviços de referencia, em horários previamente agendados, nos dias de suas consultas médicas com o infectologista dos serviços. As entrevistas foram realizadas individualmente entre maio de 2013 e maio de 2014 por um psicólogo (pesquisador responsável) e cinco estudantes de cursos de graduação, previamente treinados. Antes de iniciar a entrevista, o pesquisador responsável avaliou a aptidão cognitiva dos pacientes por meio da aplicação do Mini Exame do Estado Mental (MEEM) (Tombaugh \& McIntyre, 1992), a partir do qual três dos pacientes foram excluídos por apresentarem problemas cognitivos.

\section{Análise dos dados}

Os itens e os escores dos domínios do WHOQOL-HIV Bref foram descritos por meio de valor mínimo e máximo, média, mediana e desvio-padrão. A habilidade do instrumento em acessar todos os níveis de qualidade de vida na amostra foi verificada pela análise dos efeitos piso e teto dos seus itens. A inexistência desses efeitos foi considerada quando a porcentagem de respostas nos extremos foi inferior a 20\% (Canavarro \& Pereira, 2011). Foram também calculados os escores dos seis domínios e da qualidade de vida geral de acordo com o algoritmo construído e padronizado pela OMS, em uma escala de 4 a 20, em que os maiores escores indicam melhor qualidade de vida (WHO, 2002; Zimpel \& Fleck, 2007). A contagem de CD4 $4^{+}$foi obtida por meio da análise dos prontuários clínicos dos participantes.

Para verificar a adequação dos dados obtidos na versão em português do WHOQOL-HIV Bref à estrutura fatorial com seis fatores, realizou-se análise fatorial confirmatória. Foram utilizados como índices de qualidade do ajustamento o $\chi^{2} / g l$ (razão qui-quadrado e graus de liberdade), CFI (confirmatory fit index), GFI 
(goodness of fit index) e RMSEA (root mean square error of approximation). $\mathrm{O}$ ajustamento do modelo foi considerado adequado para valores do $\chi^{2} / \mathrm{gl}$ inferiores a 5, CFI e GFI superiores a 0,90 e de RMSEA inferiores a 0,10 (Marôco, 2010).

A validade convergente do instrumento foi avaliada pelas correlações entre os escores dos domínios e pelas correlações entre os itens de cada domínio (Marôco, 2010). Para tal, foi adotado o coeficiente de correlação de Spearman, considerando-se significativos os coeficientes com valor de $p \leq 0,05$. A validade discriminante foi estimada pelo teste U de Mann-Whitney, visto que a os escores da qualidade de vida não apresentaram distribuição normal. Compararam-se os escores dos domínios do WHOQOL-HIV Bref entre subgrupos de pacientes que hipoteticamente poderiam apresentar níveis diferentes de qualidade de vida: contagem de células $\mathrm{CD}^{+}$, presença de febre, perda de peso e presença de diarréia, uso de drogas ilícitas e classe econômica. Foi adotado o nível de significância $\alpha=0,05$.

A consistência interna do WHOQOL-HIV Bref foi medida pelo coeficiente alfa de Cronbach para a escala geral, para a escala geral se cada item for retirado e para cada domínio, sendo considerados adequados valores $\alpha \geq 0,70$ (Hair Jr, Black, Babin, Anderson \& Tatham, 2009). Para estimar a reprodutibilidade, o instrumento foi aplicado e reaplicado em 30 pacientes em um intervalo de quinze dias. Calculou-se o coeficiente de correlação intraclasse (CCI) dos escores dos seis domínios, sendo que valores superiores a 0,60 foram considerados adequados (Saddki et al., 2009).

A validade de critério concorrente foi verificada por meio das correlações entre os escores dos domínios do WHOQOL-HIV Bref com a auto percepção da qualidade de vida e da saúde. Para averiguação da validade de critério divergente foram utilizadas as correlações entre os escores dos domínios do WHOQOL-HIV Bref e os escores do Inventário de Depressão de Beck (BDI) (Beck \& Steer, 1996), uma vez que há a hipótese de que os indivíduos com alto escores de qualidade de vida apresentem baixos escores de sintomas depressivos. Desta forma, apesar de serem construtos diferentes, apresentam-se correlacionados inversamente. Todas as correlações foram avaliadas pelo coeficiente de correlação de Spearman, sendo consideradas significativas aquelas com valor de $\mathrm{p} \leq 0,05$.

O software EpiData 3.1 foi usado para a dupla digitação e conferência dos dados, e o programa Statistical Package for Social Science, (SPSS) versão 18.0 para a análise estatística dos dados.

\section{Resultados}

As medidas descritivas, de consistência interna e reprodutibilidade dos itens e domínios do WHOQOL-HIV-Bref estão apresentadas na Tabela 1.

Conforme os dados da Tabela 1 pode-se observar que o efeito piso não foi constatado nos itens avaliados e o efeito teto foi inexistente em 21 itens do instrumento. As médias dos escores dos domínios do WHOQOL-HIV-Bref variaram entre 13,1 a 15,4, para os domínios ambiente e espiritualidade, respectivamente. O escore médio da qualidade de vida geral foi de 14,7.

As medidas de consistência interna da escala global do WHOQOL-HIV Bref apresentaram valores de coeficientes alfa de Cronbach igual a 0,93. O coeficiente alfa de Cronbach variou de 0,60 a 0,76 entre as seis dimensões e o escore da qualidade de vida geral; e quando cada item foi retirado da análise esse coeficiente apresentou valores superiores a 0,92. Quanto à reprodutibilidade, o coeficiente de correlação intra-classe para os domínios variou entre 0,90 a 0,98 , indicando excelente concordância entre os valores dos escores obtidos nos dois momentos avaliados (Tabela 1).

A figura 01 apresenta a adequação dos dados para a estrutura fatorial com seis fatores do WHOQOL-HIV BREF por meio da análise fatorial confirmatória.

A maioria dos itens (79,3\%) apresentou pesos fatoriais maiores que 0,50 . As correlações entre os domínios do instrumento variaram de 0,67 a 1,06 (Figura 1). Os indicadores de ajuste do modelo aos dados apontaram para a pertinência da estrutura fatorial com seis fatores $\left(\chi^{2} / \mathrm{df}=1,77\right.$; CFI $=0,89 ; \mathrm{GFI}=0,85$; RMSEA $=$ 0,058) do WHOQOL-HIV Bref.

Os resultados da validade de construto convergente com os dados da correlação entre domínios e da correlação entre os itens nos domínios do WHOQOL-HIV Bref estão demonstrados na Tabela 2.

$\mathrm{Na}$ análise das correlações entre os seis domínios do WHOQOL-HIV Bref, observou-se que todas as correlações foram estatisticamente significativas ( $p$ $\leq 0,05)$. As correlações mais elevadas registraram-se entre o domínio físico e os domínios psicológico $(\mathrm{r}=$ $0,71)$ e nível de independência $(r=0,69)$, bem como entre os domínios psicológico e ambiente $(\mathrm{r}=0,65)$. $\mathrm{Na}$ análise de correlações dos itens que compõem cada domínio observaram-se correlações significativas ( $\mathrm{p} \leq$ $0,05)$, exceto entre os itens ambiente físico versus transporte e entre os itens saúde e assistência social versus ambiente físico do domínio meio ambiente (Tabela 2). A correlação entre cada item do instrumento e seu 
Tabela 1.

Estatística descritiva, medidas de consistência interna e reprodutibilidade do Whoqol-HIV-Bref. Montes Claros, MG, Brasil, 2014

\begin{tabular}{|c|c|c|c|c|c|c|c|c|c|}
\hline Item & $\begin{array}{l}\text { Min/ } \\
\text { Max* }\end{array}$ & Média & Med $\dagger$ & $\mathrm{Dp} \ddagger$ & $\begin{array}{c}\text { EPiS } \\
(\%)\end{array}$ & $\begin{array}{c}\text { ETe } \\
|| \\
(\%)\end{array}$ & $\begin{array}{c}\alpha \text { de } \\
\text { Cronbach } \uparrow\end{array}$ & $\begin{array}{c}\alpha \text { de } \\
\text { Cronbach }\end{array}$ & $\mathrm{CCI}^{* *}$ \\
\hline - Domínio Físico & $5,0-20,0$ & 14,4 & 15,0 & 3,4 & & & & 0,70 & 0,90 \\
\hline $\begin{array}{l}\text { Dor e } \\
\text { desconforto }\end{array}$ & $1,0-5,0$ & 3,9 & 4,0 & 1,2 & 4,0 & 41,2 & 0,93 & & \\
\hline Energia e fadiga & $1,0-5,0$ & 3,4 & 4,0 & 1,0 & 3,5 & 10,6 & 0,92 & & \\
\hline Sono e repouso & $1,0-5,0$ & 3,5 & 4,0 & 1,1 & 7,1 & 13,3 & 0,93 & & \\
\hline Sintomas de HIV & $1,0-5,0$ & 3,6 & 4,0 & 1,4 & 6,2 & 40,3 & 0,93 & & \\
\hline $\begin{array}{l}\text { - Domínio } \\
\text { Psicológico }\end{array}$ & $5,6-20,0$ & 14,6 & 15,2 & 2,8 & & & & 0,76 & 0,98 \\
\hline $\begin{array}{l}\text { Sentimentos } \\
\text { positivos }\end{array}$ & $1,0-5,0$ & 3,4 & 4,0 & 1,0 & 5,3 & 9,3 & 0,93 & & \\
\hline Concentração & $1,0-5,0$ & 3,21 & 3,0 & 0,9 & 3,1 & 7,1 & 0,93 & & \\
\hline $\begin{array}{l}\text { Imagem corporal } \\
\text { e aparência }\end{array}$ & $1,0-5,0$ & 3,84 & 4,0 & 1,0 & 3,1 & 2,7 & 0,93 & & \\
\hline Auto estima & $1,0-5,0$ & 3,8 & 4,0 & 0,9 & 3,5 & 18,1 & 0,93 & & \\
\hline $\begin{array}{l}\text { Sentimentos } \\
\text { negativos }\end{array}$ & $1,0-5,0$ & 3,9 & 4,0 & 1,0 & 5,3 & 29,6 & 0,93 & & \\
\hline $\begin{array}{l}\text { - Domínio Nível } \\
\text { de independência }\end{array}$ & $6,0-20,0$ & 13,9 & 14,0 & 3,1 & & & & 0,72 & 0,90 \\
\hline $\begin{array}{l}\text { Dependência de } \\
\text { medicação }\end{array}$ & $1,0-5,0$ & 2,9 & 3,0 & 1,2 & 10,6 & 12,8 & 0,93 & & \\
\hline Mobilidade & $1,0-5,0$ & 3,8 & 4,0 & 1,0 & 2,7 & 26,5 & 0,92 & & \\
\hline $\begin{array}{l}\text { Atividades da } \\
\text { vida diária }\end{array}$ & $1,0-5,0$ & 3,6 & 4,0 & 1,0 & 3,5 & 14,2 & 0,92 & & \\
\hline $\begin{array}{l}\text { Capacidade de } \\
\text { trabalho }\end{array}$ & $1,0-5,0$ & 3,5 & 4,0 & 1,1 & 4,9 & 14,2 & 0,92 & & \\
\hline $\begin{array}{l}\text { - Domínio } \\
\text { Relações sociais }\end{array}$ & $4,0-20,0$ & 14,6 & 15,0 & 3,0 & & & & 0,76 & 0,91 \\
\hline Inclusão social & $1,0-5,0$ & 3,6 & 4,0 & 0,9 & 12,2 & 13,7 & 0,93 & & \\
\hline Relações pessoais & $1,0-5,0$ & 3,8 & 4,0 & 0,9 & 2,2 & 20,8 & 0,93 & & \\
\hline Atividade sexual & $1,0-5,0$ & 3,53 & 4,0 & 1,0 & 5,8 & 11,9 & 0,93 & & \\
\hline Apoio social & $1,0-5,0$ & 3,7 & 4,00 & 1,0 & 6,2 & 19,5 & 0,93 & & \\
\hline $\begin{array}{l}\text { - Domínio } \\
\text { Ambiente }\end{array}$ & $6,0-19,5$ & 13,1 & 13,5 & 2,5 & & & & 0,76 & 0,94 \\
\hline $\begin{array}{l}\text { Segurança física e } \\
\text { segurança }\end{array}$ & $1,0-5,0$ & 3,4 & 4,0 & 0,9 & 3,5 & 7,1 & 0,93 & & \\
\hline Ambiente físico & $1,0-5,0$ & 3,3 & 3,0 & 0,94 & 3,5 & 7,1 & 0,93 & & \\
\hline $\begin{array}{l}\text { Recursos } \\
\text { financeiros }\end{array}$ & $1,0-5,0$ & 2,6 & 3,0 & 0,9 & 13,7 & 4,0 & 0,93 & & \\
\hline $\begin{array}{l}\text { Novas } \\
\text { informações e } \\
\text { habilidades }\end{array}$ & $1,0-5,0$ & 3,4 & 3,0 & 1,0 & 4,4 & 12,4 & 0,93 & & \\
\hline
\end{tabular}


Tabela 1.

Estatística descritiva, medidas de consistência interna e reprodutibilidade do Whoqol-HIV-Bref. Montes Claros, MG, Brasil, 2014 (Continuation)

\begin{tabular}{|c|c|c|c|c|c|c|c|c|c|}
\hline Item & $\begin{array}{l}\text { Min/ } \\
\text { Max* }\end{array}$ & Média & Med $\dagger$ & Dpł & $\begin{array}{l}\text { EPi\ } \\
(\%)\end{array}$ & $\begin{array}{l}\text { ETe } \\
|| \\
(\%)\end{array}$ & $\begin{array}{c}\alpha \mathrm{de} \\
\text { Cronbach }\end{array}$ & $\begin{array}{c}\alpha \text { de } \\
\text { Cronbach }\end{array}$ & $\mathrm{CCI}^{* *}$ \\
\hline Recreação e lazer & $1,0-5,0$ & 2,9 & 3,0 & 1,1 & 11,5 & 6,2 & 0,93 & & \\
\hline $\begin{array}{l}\text { Ambiente } \\
\text { doméstico }\end{array}$ & $1,0-5,0$ & 3,6 & 4,0 & 1,1 & 6,2 & 18,1 & 0,93 & & \\
\hline $\begin{array}{l}\text { Saúde e } \\
\text { assistência social }\end{array}$ & $1,0-5,0$ & 3,7 & 4,0 & 1,0 & 4,0 & 19,5 & 0,93 & & \\
\hline Transporte & $1,0-5,0$ & 3,4 & 4,0 & 1,1 & 7,5 & 11,9 & 0,93 & & \\
\hline $\begin{array}{l}\text { - Domínio } \\
\text { Espiritualidade }\end{array}$ & $5,0-20,0$ & 15,4 & 16,0 & 3,5 & & & & 0,60 & 0,90 \\
\hline $\begin{array}{l}\text { Espiritualidade } \\
\text { (significado da } \\
\text { vida) }\end{array}$ & $1,0-5,0$ & 3,8 & 4,0 & 1,0 & 4,4 & 19,9 & 0,93 & & \\
\hline Perdão e culpa & $1,0-5,0$ & 3,7 & 5,0 & 1,5 & 7,1 & 52,7 & 0,93 & & \\
\hline $\begin{array}{l}\text { Preocupações } \\
\text { sobre o futuro }\end{array}$ & $1,0-5,0$ & 3,9 & 5,0 & 1,3 & 5,3 & 50,9 & 0,93 & & \\
\hline $\begin{array}{l}\text { A morte e o } \\
\text { morrer }\end{array}$ & $1,0-5,0$ & 4,0 & 5,0 & 1,4 & 6,2 & 60,2 & 0,93 & & \\
\hline $\begin{array}{l}\text { Qualidade de } \\
\text { Vida Geral }\end{array}$ & $4,0-20,0$ & 14,7 & 16,0 & 3,0 & $-0,8$ & 1,1 & & 0,93 & 0,94 \\
\hline $\begin{array}{l}\text { Como avalia a } \\
\text { sua qualidade de } \\
\text { vida? }\end{array}$ & $1,0-5,0$ & 3,7 & 4,0 & 0,8 & 0,9 & 14,6 & 0,93 & & \\
\hline $\begin{array}{l}\text { Até que ponto } \\
\text { está satisfeito(a) } \\
\text { com a sua saúde? }\end{array}$ & $1,0-5,0$ & 3,6 & 4,0 & 0,8 & 2,2 & 8,8 & 0,93 & & \\
\hline
\end{tabular}

*Valor mínimo e máximo; †Mediana †Desvio padrão; $\int$ Efeito piso; | | Efeito teto; $\uparrow$ Coeficiente alfa de Cronbach (indica exclusão do item da escala); ${ }^{* *}$ coeficiente de correlação intraclasse.

escore total foi positiva com valores superiores a 0,31 , exceto para um dos itens do domínio Espiritualidade que aborda a preocupação do indivíduo com a morte $(\mathrm{r}=0,22)$.

Está apresentada na Tabela 3 a validade de construto discriminante com as comparações dos escores dos domínios do WHOQOL-HIV Bref entre subgrupos de pacientes segundo as variáveis: contagem de células $\mathrm{CD}^{+}$, presença de febre, perda de peso, episódio de diarréia, uso de drogas ilícitas, classe econômica.

Constataram-se diferenças significativas $(\mathrm{p} \leq 0,05)$ entre o escore de qualidade de vida geral e todas as variáveis analisadas. Observou-se também que a variável classe econômica apresentou associação significante $(p \leq 0,05)$ entre os escores de todos os seis domínios (Tabela 3).

As validades de critério concorrente e divergente do WHOQOL -HIV-Bref podem ser verificadas na Tabela 4.

Foi verificada associação estaticamente significativa $(p \leq 0,05)$ entre os escores dos domínios do WHOQOL-HIV Bref com a autopercepção da qualidade de vida e da saúde, confirmando a validade concorrente. Registrou-se que todos os domínios do WHOQOL-HIV Bref apresentaram correlação negativa e significativa (variando de $-0,70$ a $-0,50$ ) com o escore da escala de depressão (BDI), demonstrando validade divergente do instrumento (Tabela 4). 


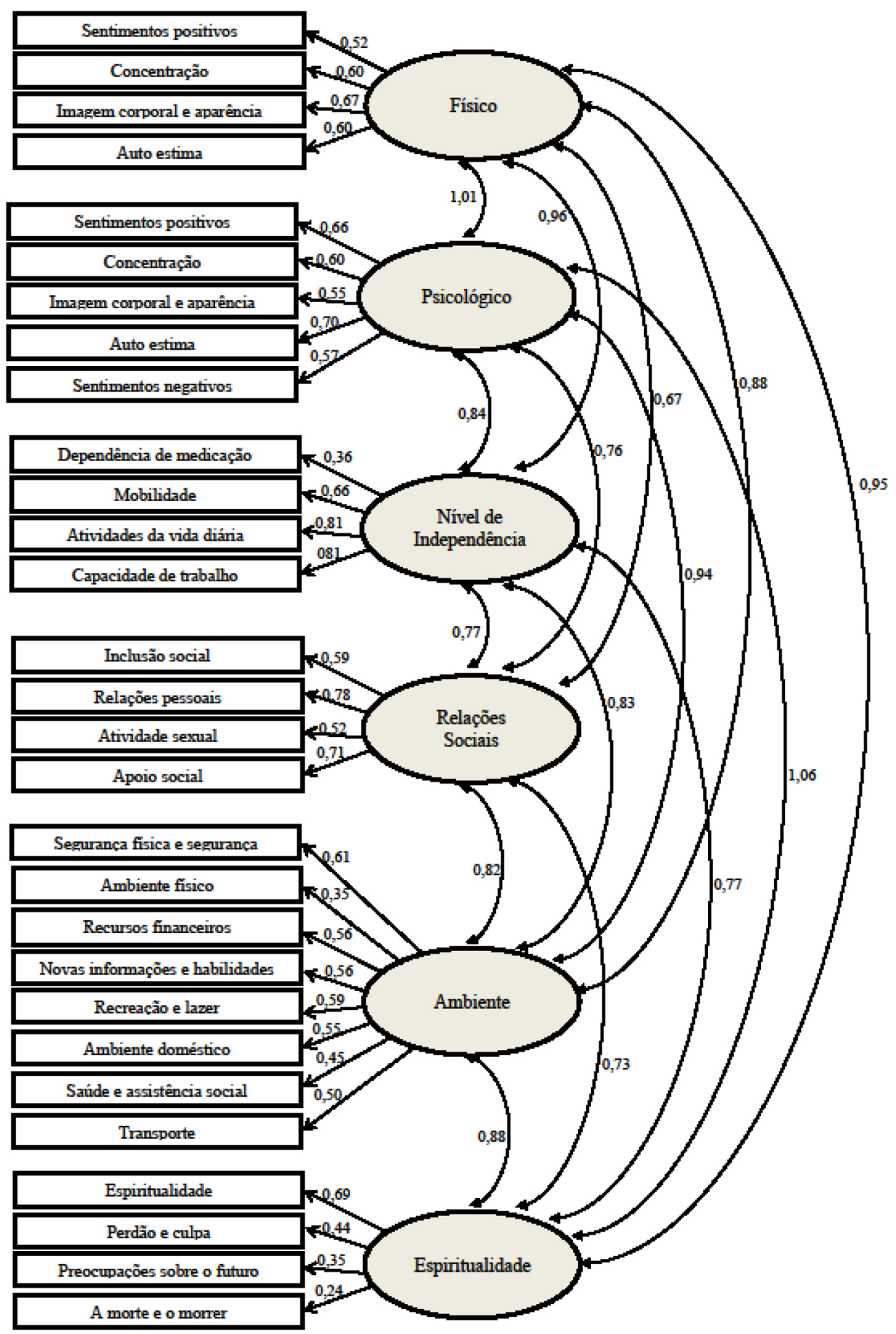

Figura 1. Estrutura do WHOQOL-HIV BREF baseada na análise fatorial confirmatória.

\section{Discussão}

A avaliação da qualidade de vida relacionada à saúde (QVRS) de pessoas infectadas pelo HIV torna-se cada vez mais importante diante do aumento da sobrevivência destes indivíduos, sendo extremamente relevante para avaliar os impactos das intervenções relacionadas ao HIV. No entanto, há um número limitado de instrumentos de avaliação para este fim (Meemon, Paek, Yenchai \& Wan, 2016). 
Tabela 2.

Validade de construto convergente do WHOQOL-HIV Bref. Montes Claros, MG, Brasil, 2014.

Correlação entre domínios

Correlação entre itens nos domínios

\begin{tabular}{|c|c|c|c|c|c|c|}
\hline Domínio & FIS $†$ & PSI & INDS & SOC || & AMB & Min/Maxt+ \\
\hline Físico (FIS) † & & & & & & $0,29 / 0,49$ \\
\hline Psicológico (PSI) $\ddagger$ & $0,71 *$ & & & & & $0,29 / 0,45$ \\
\hline Independência (IND) S & $0,70^{*}$ & $0,63^{*}$ & & & & $0,21 / 0,73$ \\
\hline Relações sociais (SOC) || & $0,49 *$ & $0,61 *$ & $0,52 *$ & & & $0,30 / 0,63$ \\
\hline Ambiente (AMB) & $0,58^{*}$ & $0,60^{*}$ & $0,64^{*}$ & $0,63^{*}$ & & $0,14 / 0,56$ \\
\hline Espiritualidade (ESP) ** & $0,52 *$ & $0,55^{*}$ & $0,37 *$ & $0,38^{*}$ & $0,42^{*}$ & $0,15 / 0,39$ \\
\hline
\end{tabular}

*Correlação positiva pelo teste de Spearman, $\mathrm{p} \leq 0,05$; † Domínios Físico (FIS); †Psicológico (PSI); \ Independência (IND); || Relações sociais (SOC); - Ambiente (AMB); **Espiritualidade (ESP);†Min/Max = valor mínimo e máximo obtido entre as correlações dos itens no domínio.

Tabela 3.

Validade de construto discriminante do WHOQOL -HIV-Bref. Montes Claros, MG, Brasil, 2014

\begin{tabular}{|c|c|c|c|c|c|c|c|}
\hline \multirow{2}{*}{ Característica do subgrupo } & \multicolumn{7}{|c|}{ Média (desvio-padrão) } \\
\hline & FIS* & PSI $\dagger$ & IND & SOCS & $\mathrm{AMB}||$ & ESPq & QVG** \\
\hline \multicolumn{8}{|l|}{ Contagem CD4+ } \\
\hline$<500$ & $14,1(3,1)$ & $14,7(2,7)$ & $13,8(2,8)$ & $14,6(2,9)$ & $13,0(2,7)$ & $14,3(3,1)$ & $14,3(3,1)$ \\
\hline$>500$ & $14,6(3,6)$ & $14,5(2,9)$ & $14,0(3,2)$ & $14,7(3,0)$ & $13,2(2,3)$ & $15,0(2,9)$ & $15,0(2,9)$ \\
\hline Valor de $\mathrm{p}$ & 0,236 & 0,722 & 0,230 & 0,743 & 0,470 & 0,609 & 0,026 \\
\hline \multicolumn{8}{|l|}{ Febre } \\
\hline Não & $14,8(3,2)$ & $14,8(2,7)$ & $14,2(2,1)$ & $14,8(2,8)$ & $13,3(2,4)$ & $15,7(3,1$ & $14,8(3,0)$ \\
\hline Sim & $11,4(3,5)$ & $13,0(3,7)$ & $11,5(3,1)$ & $13,3(3,1)$ & $11,6(2,9)$ & $13,2(4,8)$ & $13,2(2,8)$ \\
\hline Valor de $\mathrm{p}$ & $<0,001$ & 0,042 & $<0,001$ & 0,143 & 0,020 & 0,019 & 0,009 \\
\hline \multicolumn{8}{|l|}{ Perda de Peso } \\
\hline Não & $14,6(3,3)$ & $14,8(2,9)$ & $14,9(3,0)$ & $14,8(2,9)$ & $13,3(2,5)$ & $15,6(3,4)$ & $14,9(2,9)$ \\
\hline Sim & $12,8(3,5)$ & $12,9(3,4)$ & $12,6(2,9)$ & $13,8(3,3)$ & $12,0(2,6)$ & $14,4(4,6)$ & $12,7(3,1)$ \\
\hline Valor de $\mathrm{p}$ & 0,026 & 0,026 & 0,030 & 0,377 & 0,063 & 0,329 & 0,002 \\
\hline \multicolumn{8}{|l|}{ Diarréia } \\
\hline Não & $14,6(3,3)$ & $14,7(2,7)$ & $14,1(2,1)$ & $14,8(2,9)$ & $13,32,5)$ & $15,6(3,5)$ & $14,9(2,9)$ \\
\hline Sim & $13,1(4,0)$ & $13,9(3,1)$ & $12,8(3,5)$ & $13,7(3,4)$ & $12,0(2,6)$ & $14,3(3,4)$ & $13,4(3,5)$ \\
\hline Valor de $\mathrm{p}$ & 0,053 & 0,022 & 0,053 & 0,090 & 0,015 & 0,034 & 0,018 \\
\hline \multicolumn{8}{|l|}{ Consumo de drogas ilícitas } \\
\hline Não & $14,6(3,3)$ & $14,7(2,7)$ & $14,1(2,1)$ & $14,8(2,9)$ & $13,3(2,4)$ & $15,5(3,5)$ & $14,9(2,1)$ \\
\hline Sim & $12,9(3,9)$ & $13,9(3,2)$ & $12,8(3,5)$ & $13,7(3,2)$ & $12,3(2,8)$ & $15,3(3,5)$ & $13,7(3,2)$ \\
\hline Valor de $\mathrm{p}$ & 0,020 & 0,178 & 0,101 & 0,027 & 0,028 & 0,780 & 0,038 \\
\hline \multicolumn{8}{|l|}{ Classe econômica } \\
\hline $\mathrm{B} / \mathrm{C}$ & $15,3(3,1)$ & $15,1(2,8)$ & $14,5(3,1)$ & $14,9(3,1)$ & $13,6(2,4)$ & $16,3(3,2)$ & $15,3(3,0)$ \\
\hline $\mathrm{D}$ & $12,8(3,2)$ & $13,8(2,7)$ & $12,1(2,8)$ & $14,2(2,7)$ & $12,4(2,5)$ & $14,0(3,5)$ & $13,7(2,8)$ \\
\hline Valor de $\mathrm{p}$ & $<0,001$ & 0,001 & $<0,001$ & 0,046 & 0,002 & $<0,001$ & $<0,001$ \\
\hline
\end{tabular}

* Domínios: Físico (FIS); †Psicológico (PSI); ‡ Independência (IND); \Relações sociais (SOC); || Ambiente (AMB); đEspiritualidade (ESP); **Qualidade de vida geral (QV). 
Tabela 4.

Validade de critério do WHOQOL -HIV-Bref. Montes Claros, MG, Brasil, 2014

\begin{tabular}{lcccccc}
\hline Parâmetro & FIS† & PSI $\neq$ & IND $\int$ & SOC|| & AMB & ESP** \\
\hline Validade Concorrente & & & & & & \\
Autopercepção da QV †t & $0,53^{*}$ & $0,54^{*}$ & $0,56^{*}$ & $0,47^{*}$ & $0,54^{*}$ & $0,35^{*}$ \\
Autopercepção da Saúde & $0,48^{*}$ & $0,50^{*}$ & $0,50^{*}$ & $0,41^{*}$ & $0,48^{*}$ & $0,29^{*}$ \\
Validade Divergente & & & & & & \\
Escala de Depressão de Beck & $-0,62^{*}$ & $-0,70^{*}$ & $-0,59 *$ & $-0,50^{*}$ & $-0,53^{*}$ & $-0,50^{*}$ \\
\hline
\end{tabular}

*Correlação significativa $\mathrm{p} \leq 0,05 ; \nmid$ Domínios Físico (FIS); †Psicológico (PSI); \ Independência (IND); || Relações sociais (SOC); || Ambiente (AMB); ** Espiritualidade (ESP) ;† Autopercepção da Qualidade de Vida.

O WHOQOL-HIV Bref avalia a qualidade de vida com base em aspectos específicos de pessoas vivendo com HIV/AIDS e tem se mostrado válido e confiável para esse fim (Meemon et al., 2016; Pereira, Martins, Alves \& Canavarro, 2014; Salehi, Niroumand, Erfanian, Sajjadi \& Dadgarmoghaddam, 2016; Zhu, Liu \& Qu, 2017). A análise das propriedades psicométricas deste instrumento é fundamental devido os desafios para a interpretação das questões diante das diversidades culturais (Meemon et al., 2016).

No presente estudo, o instrumento mostrou-se capaz de avaliar a qualidade de vida na amostra, pois não foi detectado efeito piso nos itens avaliados e efeito teto em 21 dos 29 itens avaliados. Isto mostra boa sensibilidade do instrumento, confirmando estudos internacionais (WHO, 2002; 2003; Zhu et al, 2017).

O instrumento WHOQOL-HIV Bref apresentou validade relacionada com o construto, evidenciada pela validade fatorial, por meio da análise fatorial confirmatória, validade convergente e validade discriminante (Maroco, 2010). Os resultados da análise fatorial confirmatória do WHOQOL-HIV BREF corroboram que a estrutura original de seis domínios é adequada, o que é consistente com outros estudos que realizaram a validade fatorial deste instrumento (Pereira et al., 2014; Tesfaye et al, 2016; Zhu et al., 2017;). Verificou-se, que a o item morte e morrer apresentou a correlação mais fraca do domínio Espiritualidade enquanto que no estudo realizado com a população chinesa foi o item espiritualidade (significado da vida) (Zhu et al., 2017). Os autores do estudo supracitado sugeriram a modificação deste item para o domínio Psicológico (Zhu et al., 2017). Todavia, na presente investigação a sua correlação com o domínio espiritualidade mostrou-se satisfatória $(\lambda=0,69)$.
A validade convergente do instrumento foi verificada pela análise das correlações entre os domínios e entre os itens de cada domínio. Os significativos valores dos coeficientes de correlações entre os seis domínios e entre os itens de cada domínio sugeriram adequada validade convergente, isto é, o construto estudado se correlaciona positiva e significativamente com outros construtos teoricamente paralelos (os domínios) e que os itens que constituem o construto apresentam correlação positiva elevadas entre si (Maroco, 2010). A validade convergente deste instrumento também foi demonstrada em estudos com pessoas vivendo com HIV/AIDS, portugueses (Canavarro \& Pereira, 2011), nigerianos (Akinboro et al., 2014) e chineses (Zhu et al., 2017).

Neste estudo foi observada a presença da validade discriminante do WHOQOL-HIV Bref, também verificada em estudos anteriores internacionais (Canavarro \& Pereira, 2011; Meemon et al., 2016; Pereira et al., 2014; WHO, 2003, Zhu et al., 2017). O instrumento mostrou-se eficaz em predizer a percepção dos sujeitos estudados, em escores, sobre a qualidade de vida geral e em relação à grande parte dos domínios considerados. Desta forma, discriminou a resposta dos sujeitos dentro de subgrupos formados com base na contagem de células $\mathrm{CD}^{+}$, presença de febre, perda de peso, episódio de diarreia, uso de drogas ilícitas e classe econômica.

Em estudo realizado com 329 pacientes tailandeses não foi encontrada diferença significativa na QVRS entre grupos com diferentes contagens de células $\mathrm{CD} 4^{+}$. O subgrupo com história de infecção oportunista apresentou uma maior QVRS em relação àqueles na fase de latência (Meemon et al., 2016). Pesquisa realizada em três províncias chinesas mostrou que indivíduos com menor contagem de células $\mathrm{CD} 4^{+}$tiveram escores mais baixos e estatisticamente significantes para todos os 
domínios. Os participantes sintomáticos apresentaram escores significativamente menores que os participantes assintomáticos nos domínios físico, psicológico e de independência (Zhu et al., 2017). Em investigação com pacientes portugueses a qualidade de vida geral $(\mathrm{QV})$, os domínios físico e de independência discriminaram bem os sujeitos considerando o estágio do HIV. Porém, nenhum dos domínios foi significativamente diferente de acordo com os subgrupos de contagem de células $\mathrm{CD}^{+}$(Pereira et al., 2014).

Nesta investigação, foram observadas maiores escores da QV nos indivíduos com melhores condições clínicas. Destaca-se a variável classe econômica que apresentou diferença estatística em todos os domínios, o que pode ser explicado em parte pela sua influência no acesso aos bens de consumo e serviços que podem repercutir nas formas de pensar a qualidade de vida (Hipolito et al., 2017).

No que se refere à confiabilidade do WHOQOL-HIV Bref, por meio da análise da consistência interna, o instrumento obteve valores satisfatórios, indicando homogeneidade dos itens que o compõe, o que concorda com outros estudos (Akinboro et al., 2014; Pereira et al., 2014, Salehi et al., 2016; WHO, 2003; Zhu et al., 2017). Os valores observados para o coeficiente alfa de Cronbach se item for retirado não se apresentaram superiores ao da escala geral $(\alpha=0,93)$. Portanto, os valores observados para o coeficiente alfa de Cronbach sugerem que nenhum dos itens poderá afetar substancialmente a confiabilidade se forem retirados. Em relação aos domínios, a consistência interna mostrou-se satisfatória (alfa de Cronbach > 0,70), exceto no domínio Espiritualidade, o que foi também observado em outros estudos (Costa \& Oliveira, 2013; Tesfaye et al., 2016; WHO, 2003; Zhu et al., 2017). Já, o estudo de validação da versão persa do WHOQOL-HIV Bref apresentou escores de alfa de Cronbach acima de 0,7 para todas as dimensões (Salehi et al., 2016).

Pesquisadores já haviam sugerido que o conteúdo e o tamanho do domínio da espiritualidade poderiam resultar em menor confiabilidade (Saddki et al., 2009) e deve ser reconhecido como uma limitação do instrumento (Tesfaye et al., 2016). Este achado pode ser decorrente de o domínio da espiritualidade comportar questões que envolvem um aspecto mais filosófico como a percepção de sentido da vida, futuro, opinião sobre a morte e relações com divindade. Todavia, não se pode negar que a espiritualidade é um fator importante na percepção da qualidade de vida e pode auxiliar no enfrentamento da vivência de pessoas que vivem com HIV (Hipolito et al., 2017).
No presente estudo, a maioria dos valores observados para as correlações entre cada item e o escore total do instrumento foi satisfatória $(r \geq 0,30)$, semelhante ao observado em estudo prévio (Salehi et al., 2016). A única exceção foi o item do domínio Espiritualidade que inqueria sobre a preocupação do indivíduo com a morte e o futuro.

Com relação à reprodutibilidade do instrumento foram observados valores de correlação intraclasse adequados $(\mathrm{CCI}>0,60)$ em todos os domínios quando esse foi aplicado de forma repetida, o que indica a existência de reprodutibilidade adequada do WHOQOL-HIV Bref no grupo de pacientes pesquisados e pressupõe que as respostas dadas em diferentes momentos são confiáveis. Estes achados estão em concordância com estudos que verificaram a presença de reprodutibilidade na versão francesa do WHOQOL-HIV (Reychler et al., 2013) e na versão chinesa do WHOQOL-HIV Bref (Zhu et al., 2017).

A validade concorrente do instrumento foi verificada por meio da avaliação das correlações entre os escores dos domínios do WHOQOL-HIV Bref com à autopercepção da qualidade de vida geral e da saúde. Os resultados apontaram para correlações positivas e significativas, cujos coeficientes variaram de 0,29 a 0,48 , que demonstram a existência de validade concorrente adequada do questionário nesse grupo de pacientes. De forma semelhante, estudo realizado na República Islâmica do Irã observou que a correlação entre cada domínio do instrumento e a qualidade de vida geral foi considerada excelente (Salehi et al., 2016). A validade concorrente também foi demonstrada em estudo realizado na China em que todos os domínios foram significativamente correlacionados com o questionário de qualidade de vida 36-item form constructed to survey bealth status (SF-36) (Zhu et al., 2017).

A validade divergente foi demonstrada neste estudo, o que está em consonância com estudos prévios (Akinboro et al., 2014; WHO, 2003). Todos os domínios do WHOQOL-HIV Bref apresentaram coeficientes de correlação inversa significativos, que variou de $-0,70$ a $-0,50$, com o Inventário de Depressão de Beck, de forma que os maiores escores na medida de qualidade de vida foram associados aos menores escores na medida de depressão. Observou-se que o domínio Psicológico é o que melhor se correlaciona com o escore total do BDI. Este achado é coerente com a proposição do domínio que é o de avaliar os aspectos psicológicos envolvidos na qualidade de vida em que facetas como "sentimentos negativos", "autoestima" e "concentração" são avaliados. 


\section{Considerações Finais}

O WHOQOL-HIV Bref apresentou-se como um instrumento válido e confiável para medir a qualidade de vida de pessoas com HIV/AIDS, demonstradas pela validade de construto (validade convergente, validade discriminante e validade fatorial), análise da confiabilidade (consistência interna e reprodutibilidade) e análise de critérios (concorrente e divergente). Apenas o domínio Espiritualidade apresentou valor inferior ao adequado na análise de consistência interna. Estes achados oferecem subsídios para o uso do WHOQOL-HIV BREF como uma medida da QV entre as pessoas que vivem com HIV no norte de Minas Gerais e para estudos comparativos.

Este estudo tem como limitação o tamanho da amostra para a realização da análise fatorial confirmatória, considerando o número de parâmetros a ser estimado no modelo de medida em avaliação e a razão de entrevistas por parâmetro estimado. Além disso, na mensuração da validação concorrente utilizou-se neste estudo a variável autoperceção da qualidade de vida e da saúde. Sugerem-se o uso outros instrumentos validados de mensuração da qualidade de vida para análise desta evidência de validade. É pertinente a realização de outros estudos para avaliação da validade e confiabilidade do instrumento WHOQOL-HIV BREF em outros cenários com unidades de tratamento para pessoas com HIV devido às diferenças nas características socioculturais.

\section{Referências}

Akinboro, A. O., Akinyemi, S. O., Olaitan, P. B., Raji, A. A., Popoola, A. A., Awoyemi, O. R., \& Ayodele, O. E. (2014). Quality of life of Nigerians living with human immunodeficiency virus. The Pan African Medical Journal, 18(234), 1-11. doi: 10.11604/ pamj.2014.18.234.2816

Beck, A. T., \& Steer, R. A. (1996). Manual for the Beck Depression inventory-II. San Antonio: Psychological Corporation.

Bellini, J. M., Reis, R. K., Reinato, L. A. F., Magalhães, R. L. B., \& Gir, E. (2015). Qualidade de vida de mulheres portadoras do HIV. Acta Paulista de Enfermagem, 28(4), 350-354. doi:10.1590/1982-0194201500059

Cooper, V., Clatworthy, J., Harding, R., Whetham, J., \& Emerge Consortium. (2017). Measuring quality of life among people living with HIV: a systematic review of reviews. Health Qual Life Outcomes, 15(1):220. doi: 10.1186/s12955-017-0778-6.

Canavarro, M. C., \& Pereira, M. (2011). Avaliação da qualidade de vida na infecção por VIH/SIDA: Desenvolvimento e aplicação da versão em Português Europeu do WHOQOL-HIV-Bref. Laboratório de Psicologia, 9(1), 49-66. Recuperado de:https://estudogeral.sib.uc.pt/ bitstream/10316/20676/1/2011\%20Desenvolvimento $\% 20 \mathrm{e} \% 20$ aplica $\% \mathrm{C} 3 \% \mathrm{~A} 7 \% \mathrm{C} 3 \% \mathrm{~A} 3 \mathrm{o} \% 20$ do $\% 20$ WHOQOL-HIV-Bref.pdf

Costa, T. L., \& Oliveira, D. C. (2013). Qualidade de vida de pessoas com vírus da imunodeficiência humana e a interiorização: avaliação multidimensional. Revista de enfermagem UFPE on line, 7(10), 5866-5875. doi: 10.5205/reuol.4377-36619-1-ED.0710201305

Costa, T. L., Oliveira, D. C., \& Formozo, G. A. (2015). The health sector in social representations of HIV/Aids and quality of life of seropositive people. Escola Anna Nery, 19(3), 475-483. doi:10.5935/1414-8145.20150063

Gomes-Oliveira, M. H., Gorenstein, C., Lotufo Neto, F., Andrade, L. H., \& Wang, Y. P. Validation of the Brazilian Portuguese version of the Beck Depression Inventory-II in a community sample (2012). Rev Bras Psiquiatr., 34, 389-394. doi: dx.doi. org/10.1016/j.rbp.2012.03.005.

Hair Jr, J. F., Black, W. C., Babin, B. J., Anderson, R. E., \& Tatham, R. L. (2009). Análise multivariada de dados. (A. S. Sant'Anna, Trad.). Porto Alegre: Bookman.

Hipolito, R. L., Oliveira, D. C., Costa, T. L., Marques, S. C., Pereira, E. R., \& Gomes, A. M. T. (2017). Qualidade de vida de pessoas convivendo com $\mathrm{HIV} /$ aids: relação temporal, sociodemográfica e perceptiva da saúde. Revista Latino-Americana de Enfermagem, 25, e2874. https:/ / dx.doi.org/10.1590/1518-8345.1258.2874

UNAIDS. Joint United Nations Programme on HIV/ AIDS. UNAIDS. (2012). Decision point la francophonie: no new HIV infections, no one denied treatment. Recuperado de: http:/ / www.unaids.org/ sites/default/files/media_asset/JC2413_201210_ francophonie-summit-kinshasa_en_3.pdf

Karkashadze, E., Gates, M.A., Chkhartishvili, N., DeHovitz, J., \& Tsertsvadze, T. (2017). Assessment of quality of life in people living with HIV in Georgia. International Journal of \& STD AIDS, 28(7), 672-678. doi: 10.1177/0956462416662379 
Maroco, J. P. (2010). Análise de equações estruturais: Fundamentos teóricos software \& aplicações. Lisboa: Report Number.

Maroco, J. P., Campos, J. A. D. B., Vinagre, M. G., \& Pais-Ribeiro, J. L. (2014). Adaptação transcultural Brasil-Portugal da escala de satisfação com o suporte social para estudantes do ensino superior. Psicologia Reflexão e Crítica, 27(2), 247-256. doi:10.1590/1678-7153.201427205

Meemon, N., Paek, S. C., Yenchai, D., \& Wan, T. T. H. (2016). Application of the WHOQOL-HIV-BREF questionnaire in HIV-infected Thai patients: reliability and validity of the instrument. $J$ Assoc Nurses AIDS Care, 27, 698-708. doi: 10.1016/j. jana.2016.04.007

Oliveira, F. B. M., Queiroz, A. A. F. L. N., Sousa, Á. F. L., Moura, M. E. B., \& Reis, R. K. (2017). Orientação sexual e qualidade de vida de pessoas vivendo com HIV/aids. Revista Brasileira de Enfermagem, 70(5), 1004-1010. https://dx.doi. org/10.1590/0034-7167-2016-0420

Osei-Yeboah, J., Owiredu, W. K. B. A., Norgbe, G. K., Lokpo, S. Y., Obirikorang, C., Alote Allotey, et al. (2017). Quality of Life of People Living with HIV/AIDS in the Ho Municipality, Ghana: A Cross-Sectional Study. AIDS Research and Treatment, 2017, 1-7. doi: 10.1155/2017/6806951.

Pereira, M., Martins, A., Alves S., \& Canavarro M. C. (2014). Assessing quality of life in middle-aged and older adults with HIV: psychometric testing of the WHOQOL-HIV-Bref. Qual Life Res, 23(9), 24732479. doi:10.1007/s11136-014-0707-7

Quadros, M. C., Cordeiro, J. M., Vargas, A. M., Barros, A. M. L. M., Almeida, T. T. S. R., Senna, M. I., \& Ferreira, R. C. (2015). Functional and psychosocial impact of oral disorders and quality of life of people living with HIV/AIDS. Quality of Life Research, 24(2), 503-11. doi: 10.1007/s11136-014-0778-5.

Reychler, G., Caty, G., Vincent, A., Billo, S., \& Yombi, J. C. (2013). Validation of the French version of the World Health Organization quality of life HIV instrument. PLoS One, 8(9), e73180. doi:10.1371/ journal.pone. 0073180

Saddki, N., Noor, M. M., Norbanee, T. H., Rusli, M. A., Norzila, Z., Zaharah, S., \& Zarina, Z. A.
(2009). Validity and reliability of the Malay version of WHOQOL-HIV BREF in patients with HIV infection. AIDS care, 21(10), 1271-1278. doi: 10.1080/09540120902803216

Salehi, M., Niroumand, S., Erfanian, M. R., Sajjadi, R. B., \& Dadgarmoghaddam, M. (2016). Validation of Persian version of WHOQOL-HIV BREF questionnaire in Islamic Republic of Iran. Eastern Mediterranean Health Journal, 22(9), 647-653.

Tesfaye, M., Olsen M. F., Medhin G, Friis, H., Hanlon, C., \& Holm, L. (2016) Adaptation and validation of the short version WHOQOL-HIV in Ethiopia. Int J Ment Health Syst, 10, 29. doi:10.1186/ s13033-016-0062-x

Tombaugh, T. N., McIntyre, N. J. (1992). The minimental state examination: a comprehensive review. J Am Geriatr Soc., 40(9), 922-935.

WHO - World Health Organization. Department of Mental Health and Substance Dependence. (2002). WHOQOL-HIV instrument: users manual. Geneva: WHO. Recuperado de: http://www.who.int/mental_health/media/en/613.pdf

WHO - World Health Organization. WHOQOL HIV Group. (2003). Initial steps to developing the World Health Organization's quality of life instrument (WHOQOL) module for international assessment in HIV/AIDS. AIDS Care, 15(3), 347357. doi: 10.1080/0954012031000105405

WHO - World Health Organization Fact Sheet. (2014). Global Update on the Health Sector Response to HIV. Geneva.

Zimpel, R. R., \& Fleck, M. P. (2007). Quality of life in HIV-positive Brazilians: application and validation of the WHOQOL-HIV, Brazilian version. AIDS Care, 19(7), 923-930. doi: 10.1080/09540120701213765

Zhu, Y., Liu J., \& Qu B. (2017). Psychometric properties of the Chinese version of the WHOQOL-HIV BREF to assess quality of life among people living with HIV/AIDS: a cross-sectional study. BMJ Open, 7(8), e016382. doi: 10.1136/ bmjopen-2017-016382.

Recebido em: 02/04/2018

Reformulado em: 22/08/2018

Aprovado em: 28/09/2018 
Sobre os autores:

Marise Fagundes Silveira é Professora Doutora do Programa de Pós-Graduação em Ciências da Saúde da Universidade Estadual de Montes Claros. Montes Claros, MG, Brasil. Possui graduação em Matemática e é especialista em Estatística.

Orcid:0000-0002-8821-3160

E-mail: ciaestatistica@yahoo.com.br

Antônio Carlos Ferreira é Professor Doutor do Departamento de Ciência e Saúde Coletiva da Universidade Estadual de Montes Claros. Montes Claros, MG, Brasil. Possui graduação em Psicologia.

Orcid: 0000-0002-3274-9097

E-mail: acf452000@yahoo.com.br

Maria Fernanda Santos Figueiredo Brito é Professora Doutora do Programa de Pós-Graduação em Cuidado Primário em Saúde da Universidade Estadual de Montes Claros e do curso de medicina do centro Universitário FipMoc. Montes Claros, MG, Brasil. Possui graduação em Enfermagem.

Orcid: 0000-0001-5395-9491

E-mail:mfsfbrito@yahoo.com.br

Lucinéia de Pinho é Professora Doutora do Programa de Pós-Graduação em Cuidado Primário em Saúde da Universidade Estadual de Montes Claros e do curso de medicina das Faculdades Integradas Pitágoras. Montes Claros, MG, Brasil. Possui graduação em Nutrição.

E-mail: lucineiapinho@hotmail.com

Orcid: 0000-0002-2947-5806

Antônio Lúcio Teixeira Júnior é Professor Doutor do Departamento de Clínica Médica da Universidade Federal de Minas Gerais. Belo Horizonte, MG, Brasil. Possui formação em Medicina e é especialista em Psiquiatria.

Orcid: 0000-0002-9621-5422

E-mail: altexr@gmail.com

Mariângela Carneiro é Professora Doutora do Departamento de Parasitologia da Universidade Federal de Minas Gerais. Belo Horizonte, MG, Brasil. Possui graduação em Farmácia e Bioquímica.

Orcid: 0000-0002-9390-7714

E-mail: mariangelacarneiro@terra.com.br

Contato com os autores:

Marise Fagundes Silveira

Av. Rui Braga, s/n, Vila Mauriceia

Montes Claros-MG, Brasil

CEP: 39401-089 